\title{
"Vieja" y "nueva" clase obrera en la Patagonia Argentina: del ferrocarril a las industrias subsidiadas por el Estado
}

\author{
Gonzalo Pérez Álvarez \\ Instituto de Investigaciones Históricas y Sociales - UNP - Conicet \\ gperezalvarez@gmail.com; gonzaloperezalvarez@yahoo.com
}

\section{Introducción}

Desde 1956 el Estado argentino promovió la instalación de industrias en la región patagónica, buscando asegurar el control del territorio nacional a través del poblamiento de esta extensa región. Para ello la dictadura de Aramburu (que un año antes, en septiembre de 1955, había derrocado al gobierno constitucional de Juan Domingo Perón) eximió de impuestos a las importaciones hacia el sur del paralelo 42 (límite norte de la provincia de Chubut) como forma de "propender al desarrollo de la Patagonia". ${ }^{1}$ Las inversiones de los empresarios se concentraron en la región más cercana al límite norte, en lo que definimos como "noreste de Chubut" (donde se ubican las ciudades de Trelew, Rawson y Puerto Madryn), expresando la lógica intención de los empresarios privados de conseguir ganancias a corto plazo, y dejando sin aportes de relevancia el sur de Chubut, y Santa Cruz y Tierra del Fuego.

Las industrias se comenzaron a instalar, y esa región vio transformada su estructura económica y social, hasta alli orientada a la producción ganadera (lana y carne) y la actividad mercantil. Su tradicional eje articulador era el ferrocarril, que desde inicios del siglo XX conectaba Alto Las Plumas (en la meseta central de Chubut) con Puerto Madryn, constituyéndose como centro del circuito comercial la ciudad de Trelew. En 1961 el gobierno de Frondizi pondría punto final a la vida de este ferrocarril, al tiempo que continuaba el proyecto de instalación del modelo de "polos de desarrollo". ${ }^{2}$

Hemos formulado la hipótesis de que en esta región se construyó una

1. Texto del Decreto 10.991/56, citado en Gatica (1998).

2. Proyectos que proponen, ante la supuesta imposibilidad de un desarrollo homogéneo de las regiones atrasadas, generar desde el estado "polos" que irradiarian el

(Archivos, año IV, $\mathrm{n}^{\circ} 7$, septiembre de 2015, pp. 143-162) 
nueva clase obrera a partir de la industrialización. Esta clase obrera se constituyó integrando diversos núcleos migrantes, que se instalaron en estas ciudades al abrigo de los puestos de trabajo en expansión. Así se habría conformado una clase obrera "joven", que comenzó a desarrollar sus primeras articulaciones y experiencias en común en el marco de la industrialización subsidiada, en un periodo de la historia argentina caracterizado por la sucesión de gobiernos autoritarios.

En este trabajo profundizamos la indagación sobre esta hipótesis, intentando problematizar resultados previos, a partir de la incorporación de nuevas fuentes y de centrar nuestra atención en el desarrollo y actividad de la clase y el movimiento obrero en los años anteriores, buscando hacer hincapié en el momento de transformación, donde se entretejen el cierre del ferrocarril (base de la "vieja" clase obrera regional) y el nacimiento de las primeras fábricas textiles al amparo de la promoción industrial (ejes de la "nueva" clase obrera). Buscamos, específicamente, encontrar las principales líneas de cambio y continuidad que se hacen observables entre las experiencias y formas de organización de estos "viejos" y "nuevos" núcleos obreros.

\section{Afluentes de la historia: fuentes y métodos}

Observamos este momento de quiebre recobrando diversos insumos documentales, analizando la destrucción y/o conformación de organizaciones obreras, el desarrollo de nuevos grupos de trabajadores y los vínculos con las experiencias y tradiciones de los antiguos núcleos.

Para ello incorporamos el registro del Archivo Histórico Provincial de Chubut y de publicaciones periodísticas de la provincia. También trabajamos en la producción y recuperación de fuentes orales. Agregamos una fuente hasta el momento inédita: un conjunto de entrevistas a ex trabajadores ferroviarios realizadas a fines de la década del 80 en el marco de un proyecto del Estado nacional para un libro conmemorativo por los cien años del comienzo de la actividad ferroviaria en la región. ${ }^{3}$

Se trata de once entrevistas a trabajadores, cuyos testimonios hoy son imposibles de relevar por haber fallecido. Sus aportes sobre las características de aquella clase obrera, sus formas de organización, sus luchas y fracasos, se constituyeron en un insumo central para este artículo. Utilizar fuentes orales producidas por otros investigadores nos obligó a

crecimiento a través del encadenamiento de actividades económicas. Ver Perroux (1955) y Rougier (2007).

3. Una pequeña parte de las entrevistas fueron incorporadas en Accorinti (1989). Susana López, reconocida historiadora de nuestra región, que coordinó esas entrevistas, nos facilitó la totalidad de las grabaciones. 
profundizar los recaudos metodológicos para su tratamiento, porque en varios casos se carecía de información precisa sobre el testimoniante, las características de la entrevista, el lugar de realización, etc. También hemos incorporado nuestra propia producción de fuentes orales, con entrevistas a otros trabajadores e hijos de aquellos ferroviarios.

\section{Una mirada general del proceso histórico de la región}

En 1865 llegaron al actual noreste de Chubut inmigrantes galeses, que comenzaron a desarrollar tareas agrícolas y comerciales. Hasta allí la región era habitada únicamente por pueblos mapuche-tehuelches. Tras la ocupación por parte del ejército argentino, a través de la conquista militar, comenzó a instalarse el poder del Estado. Esta imposición generó algunos problemas con la colonia galesa (L. Jones, 1966; M. Jones, 1981), acostumbrada a una mayor autonomía, pero los colonos rápidamente se adaptaron al proceso. Ya hacia 1874 lograron una cosecha de trigo abundante, que generaba la posibilidad de comercializarlo en el mercado nacional, siendo el transporte la principal dificultad a resolver.

Se comenzaron a formular proyectos que permitiesen esa comercialización. Con la ocupación de las tierras del sur por ovinos (Bandieri, 2005), esta necesidad fue compartida por los propietarios de las estancias que comenzaban a erigirse en Patagonia. Puertos, vías férreas y caminos se hacian necesarios para asegurar la conexión de las tierras recientemente ocupadas con el mercado central del país.

Se configuró una estructura económica organizada en torno a las actividades agrícolas y ganaderas, y un conjunto de empresas locales cuyos productos cubrian la región y parte de la Patagonia sur, merced al efecto "protector" que generaba el alto costo del transporte de los bienes procedentes de la zona metropolitana.

Hacia 1940 esto comenzó a cambiar. La baja de los costos del transporte agravó la competencia con los productos agrícolas del norte, de menor precio por las diferencias de clima. Otras producciones de la región, como su fábrica de cerveza, los molinos harineros o los tambos, tampoco lograron competir con las grandes empresas nacionales. La producción ganadera ovina será la actividad primordial (y casi única) de la región hasta la instalación de los "polos de desarrollo".

El impacto de esta política se concentró en el noreste de Chubut, que también reunió la mayoría de los puestos de trabajo generados por el Estado provincial en formación. ${ }^{4}$ Al tiempo que se impulsaba este

4. Ya que Rawson fue designada como capital de Chubut, tras su provincialización 
proceso, el Estado nacional decidía, en 1961, clausurar el ferrocarril patagónico. En 1971 se creó el Parque Industrial de Trelew y se adjudicó a la empresa Aluar (Aluminio Argentino S.A.) el proyecto de una fábrica de aluminio primario a instalarse en Puerto Madryn. El proyecto de "polos de desarrollo" tendría sus décadas de auge en los 70 y 80, y fue desarticulado durante los 90, generando nuevas transformaciones sociales en la Patagonia argentina (Pérez Álvarez, 2013).

\section{La primera formación de la clase obrera: el ferrocarril}

Hemos sostenido que durante los primeros años de la industrialización subsidiada (desde fines de la década del 50) se empezó a formar una nueva clase obrera, producto del arribo de diversos contingentes migrantes. Esta clase, heterogénea y que en muchos casos no traía experiencia (Thompson, 1989) de trabajo fabril o vida urbana, se encontró con un contexto que le posibilitaba ir mejorando sus condiciones de vida en el marco del proceso de industrialización.

Consideramos que esta nueva clase no estableció vínculos fuertes con las experiencias y tradiciones previas; esto no implica que hasta allí no existieran conflictos u organización obrera, ${ }^{5}$ pero sí que la clase que se conformó no parecía haber construido lazos relevantes con la historia de organización de los trabajadores durante la primera mitad del siglo XX. Por ello el rasgo central de esta joven clase sería el de su heterogeneidad, su ausencia de tradiciones en común y su escasa experiencia colectiva en el terreno politico y sindical. Estos rasgos, junto al contexto de una industrialización dependiente de subsidios estatales, serian factores explicativos claves de dos elementos que observamos como característicos de la clase: una conflictividad moderada en lo laboral y lo político (especialmente en comparación con lo que acontecía en otras regiones del pais durante el mismo periodo), y la identificación de intereses entre estos trabajadores y los grupos dominantes del polo de desarrollo (Pérez Álvarez, 2013).

En este artículo complejizamos esa mirada, desde el aporte de los testimonios de once trabajadores ferroviarios. ${ }^{6} \mathrm{El}$ primer dato evidente es que casi todos los entrevistados habian sido, antes del ingreso al fe-

en 1957.

5. Hay conflictos relevados ya en el inicio del siglo XX (Gatica y Pérez Álvarez, 2012: 187-214).

6. Son: Cayetano Siciliano (italiano), Edgar Calderón (de La Pampa), Federico Thomas (de la colonia galesa), Gilberto Hughes (de la colonia galesa), Antonio Maliqueo (de origen mapuche), Raúl Krotevich (origen ruso y alemán), Roberto Roberts (de la 
rrocarril, trabajadores rurales o hijos de pequeños propietarios de tierras en la zona del Valle. Se expresa así un proceso concreto de proletarización y urbanización. Esa vieja clase también tenía diversos afluentes y presentaba asimismo distintas experiencias y tradiciones en su seno.

En sus testimonios aparecen dos momentos de crisis y dos de bienestar. Sobre los primeros nos cuentan que "en 1914 hay una gran crisis en Puerto Madryn, los desocupados se juntan en la plaza y piden comida al municipio" (Accorinti, 1989: 43, testimonio de Cayetano Siciliano) y, especialmente, hacia 1930, cuando con el gobierno de Justo se ingresó a una etapa muy dificil: "En el 33 todo era una miseria" (idem: 45; mismo testimonio). Juan Federico Thomas dice que "el 34 y el 35 fueron años muy crudos" (idem: 100).

Los momentos positivos fueron el primer gobierno de Yrigoyen y, especialmente, el periodo inicial de Perón. Es alli donde, según sus memorias, los derechos de los trabajadores tuvieron un avance sustancial. Esto comenzó a desvanecerse con el segundo gobierno peronista: en ese momento se inició una larga noche, que se oscureceria mucho más tras el golpe de 1955.

Para Thomas, durante el gobierno de Perón, "el obrero pudo contar con su respaldo... había muchas injusticias" (Accorinti, 1989: 106). Según Roberto Roberts, "dio todo a los ferroviarios, hasta el 52 o 53, cuando empezó a dar palos y a poner en régimen militar prohibiendo las huelgas... La mejor época fue el primer gobierno de Perón" (Accorinti, 1989: 194). ${ }^{7}$ Victorino Ibañez destacó el dolor que siguió a 1955: "En el 55 se produce el golpe y son horas tristes y dificiles, con hechos lamentables como cesantías a trabajadores de la Unión Ferroviaria y La Fraternidad y a los que eran de la CGT" (Accorinti, 1989: 269). Según el mismo entrevistado, "a mi padre en el ferrocarril lo explotaron, él trabajaba muchas horas y le pagaban una miseria...; ino le alcanzaba ni para comer! Por eso para mí el mandato de Perón fue beneficioso; el primer gobierno, porque el segundo fue distinto" (Accorinti, 1989: 204).

Años antes los trabajadores ferroviarios tuvieron un rol central en la conformación de la primera regional de la CGT, hacia 1946. José Bueno, dirigente de la Unión Ferroviaria en Puerto Madryn, impulsó la formación de la regional junto a Toribio Medina, de la Asociación

colonia galesa), Vicente Accorinti (italiano, hijo de ferroviario), Ignacio Bernárdez (sin datos), Peter Seibt (alemán) y Victorino Ibáñez (español).

7. Durante dicho gobierno Roberts pronunció un discurso, como representante de la Unión Ferroviaria, en la entrega del Barrio Ferroviario de Trelew. Destacó que la entrega de las casas era producto del gobierno justicialista, y llamó a votar el 11 de noviembre de 1951 por la lista "que encabeza nuestro líder el general Perón". Finalizó diciendo: "Viva el general Perón, Viva Evita, Viva la Unión Ferroviaria y Viva el Ferrocarril Nacional" (p. 192). 
del Personal Aeronáutico, quien fue el primer delegado regional. Eliseo Sien (dirigente de Vialidad Nacional, de la Asociación de Trabajadores del Estado) era el subdelegado; Jorge Yunes (empleado municipal) fue Tesorero; Raúl Krotevich (maquinista de La Fraternidad), protesorero; y Gilberto Hughes (por la Unión Ferroviaria), secretario de actas.

Los siguientes años se conformaron nuevas estructuras gremiales en la región, mientras en el ferrocarril se desarrollaron varias huelgas durante el segundo mandato peronista. El personal se amplió a 300 empleados, y fueron constantes los reclamos por las condiciones de trabajo, con un desempeño apegado al reglamento que, según los obreros, complicaba el servicio en una región donde los imprevistos eran constantes.

A los problemas de funcionamiento del ferrocarril se le sumaba la mayor competencia del transporte automotriz y la disminución del tránsito de barcos, procesos que restaban centralidad al tren como medio de circulación. Hacia 1957 ya se empezaron a plantear rumores sobre su posible clausura. La organización de los trabajadores se había resentido tras el golpe que dispuso la cesantía de sus principales dirigentes, aunque en 1958, tras la victoria de Frondizi, algunos de ellos fueron reincorporados.

La CGT reorganizada pasó a ser dirigida por Gilberto Hughes, en un contexto donde se presentaron diversos conflictos con las empresas y entre los mismos dirigentes sindicales, ya que tras la instalación de las primeras fábricas se integró el gremio textil a la central obrera. Comenzaba a plantearse el momento de cambio.

\section{Los ferroviarios que perdieron su tren}

El quiebre se profundizó con la clausura del ferrocarril, en 1961. El gobierno de Frondizi, junto a funcionarios del Banco Mundial, desarrollaron el "Plan Larkin" que impulsaba el cierre de ramales y el fomento del transporte automotriz. El 30 de octubre de 1961, y tras algunos paros parciales, se inició la gran huelga ferroviaria nacional, que se prolongaria por 42 dias. Para ese momento ya habian comenzado los cierres de ramales, talleres y coches comedores. Algunas seccionales (Liniers, Rosario y Tafi Viejo) iniciaron la huelga y, tras varios titubeos, la Comisión Directiva nacional decretó el paro por tiempo indetermi-

8. Tomó su nombre del general estadounidense Thomas Larkin, quién encabezaba la comisión de estudio de los transportes en Argentina. Dicha comisión sostuvo que era necesario abandonar el $32 \%$ de las vías existentes, despedir 70.000 empleados y eliminar las locomotoras a vapor. 
nado (Ortega, 2010; Scodeller, 2007; Lucita, 1999; Schneider, 2006; Cena, 2009). ${ }^{9}$

En la región no hemos registrado enfrentamientos, ya sea con las fuerzas represivas o el gobierno, hechos que fueron una constante durante la huelga en el resto del país y que sí se produjeron en San Antonio Oeste $^{10}$ (300 km al norte de Puerto Madryn). En el noreste de Chubut la huelga se realizó sin acciones en las calles, y al pretender regresar a sus puestos, una vez que el conflicto había finalizado a nivel nacional, los trabajadores se encontraron con la clausura definitiva del ramal. Peter Seibt cuenta: "Después de una huelga nos encontramos con la noticia de que se habia clausurado el ferrocarril" (Accorinti, 1989: 238).

Thomas lo desarrolla en extenso:

En octubre hubo una huelga en toda la república, desde las provincias hacia Capital Federal. Nos movilizamos hacia allá, porque no daban cumplimiento a los reclamos de los ferroviarios y todas esas cosas. La central de Buenos Aires no quería hacer paro y hubo presión desde las provincias... la central no quería hacer, tenía sus conversaciones con el gobierno. Pero apoyaban, y al mismo tiempo tuvimos una huelga de casi cuarenta días en todo el país. Ahí aprovecharon eso para provocar los cierres de ferrocarril. Siguieron funcionando acá en Patagonia los trenes de Comodoro y Deseado, estaban en el decreto de clausura pero los sostuvieron un tiempo. ${ }^{11}$

Thomas nos informa que ya habian realizado otras medidas de lucha, como una huelga en noviembre de $1960 .{ }^{12} \mathrm{Y}$ su testimonio expresa la escasa reacción colectiva ante la clausura: "El problema más grave que me tocó fue cuando se clausuró el ramal en el año 61; ¡estaba solo!, acá empezaron a indemnizar a la gente, jla gente se fue...!" (sic, original). ${ }^{13}$

Vicente Decaro, por su parte, lo recuerda asi:

Hubo paros generales en todo el país, cuarenta y tantos

9. Utilizamos, entre otros, los siguientes trabajos para reconstruir el proceso: .

10. El 24 de noviembre de 1961, los dirigentes de La Fraternidad de San Antonio Oeste fueron citados por la delegación de la policía federal a una reunión para negociar la huelga, y allí quedaron detenidos. Ante esto la población se movilizó masivamente, rodeando la seccional e interrumpiendo el tráfico, hasta obtener la libertad de los detenidos.

11. Entrevista a Juan Federico Thomas, por Susana López, 3 de agosto de 1988.

12. El diario Jornada, martes 8 de noviembre de 1960, titula "Se cumplió el paro en Trelew". No hay información de acciones en la calle.

13. Entrevista a Juan Federico Thomas, por Susana López, 3 de agosto de 1988. 
días de paro, pero nos fueron comprando con la indemnización. Cuando fuimos a trabajar, después de la huelga, nos encontramos todo clausurado. $\mathrm{Y}$ ahí armamos una comisión de la Unión Ferroviaria y La Fraternidad, fuimos tres veces a la gobernación... Estaba Galina, ${ }^{14}$ le pedimos que lo comprara la provincia (al ferrocarril), y cuando dio vueltas y nos decía que no, le pedimos armar una cooperativa, que nosotros nos hacíamos cargo... pero no hubo caso, no quería saber nada. Nunca nos quedamos nada escrito, todo charlado nomás. ${ }^{15}$

En este testimonio encontramos prácticas obreras que, en avances previos, habíamos considerado que partían de la experiencia de industrialización subsidiada. Pensamos que la centralidad que en los reclamos laborales adquirió la solicitud de los trabajadores para que el Estado provincial cubriese las actividades que el Estado nacional abandonaba tenía su origen en las características del modelo de polo de desarrollo, donde el elemento capitalista fundamental era personificado por el aparato estatal. Por ello las demandas centralmente se presentan ante el Estado, en sus diferentes instancias.

Aquí vemos que esta característica es anterior a la industrialización. Los trabajadores del ferrocarril le reclamaron a la provincia que se hiciera cargo de la empresa, o que ellos pudieran seguir trabajando como una cooperativa. Lejos de realizar acciones de conflictividad abierta, el camino elegido fue la audiencia con las autoridades públicas.

En los testimonios se registra una constante referencia al ferrocarril como símbolo de soberanía y poblamiento. Como luego se haría con la industria, la defensa del tren se intentó sostener en torno a un discurso que no planteaba el resguardo de los derechos laborales, sino el amparo de la región y de su "soberanía". Fueron escasos los apoyos de otros gremios. Esto planteó que los nuevos gremios (textiles y metalúrgicos), que ya eran la fracción más dinámica de la clase obrera regional, no estaban interesados en defender el emblema de la etapa previa a la industrialización subsidiada.

Los ferroviarios buscaron generar la movilización como ciudad o región. Pero el resto de la sociedad no los apoyó: el ferrocarril ya no era visto como factor de "progreso", y ese rol lo pasaba a cumplir el proyecto industrializador, integrado al transporte automotor y a las rutas pavimentadas que conectarian la región con el norte del país.

El reclamo, como volvió a suceder cuando comenzaron a cerrar las

14. Jorge Galina fue el primer gobernador constitucional de la Provincia del Chubut (1958-1962), miembro de la Unión Cívica Radical Intransigente, al igual que el presidente Frondizi.

15. Entrevista a Vicente Decaro, por Susana López, 28 de abril de 1988. 
fábricas subsidiadas, se concentró en el plano legal, solicitando el pago de las indemnizaciones y el posible traspaso a una entidad cooperativa. Como la indemnización fue respetada, eso llevó a que casi no se registrasen protestas. Así lo evaluaba Decaro, años después:

Cuando se cerró estaba Galina acá, Frondizi en lo nacional, y esos nos compraron con la indemnización... La indemnización nos llevó a no protestar, fue un sueldo por año de servicio y las vacaciones...

\section{Dice Thomas:}

Se nos paga una indemnización que fue importante, en eso el ferrocarril se portó. No recuerdo a cuántos sueldos correspondía pero era una cifra importante. En Trelew ya había industrias, así que algunos se sumaron a eso, otros a la usina, la municipalidad, pero no todos, muchos se fueron de la zona, especialmente los más jóvenes...

Finalmente cualquier posibilidad de reapertura quedó anulada el 8 de junio de 1964, cuando se produjo un incendio que arrasó las instalaciones ferroviarias que habian quedado en Trelew. El hecho fue intencional, pero nunca se dio con los culpables, pese a que la mayoría señalaba que los interesados en sepultar definitivamente el tren eran los propietarios del transporte automotor, que así pasaba a ser el único medio de circulación.

Destacamos, nuevamente, la ausencia de hechos de conflictividad abierta. El cierre del ferrocarril se registró como un acontecimiento que produjo dolor y tristeza, y hasta como un ataque a la región y su identidad, pero sin que existiesen manifestaciones. De hecho la causa de su clausura (y no de otros ramales cuyo final también se proyectaba) ${ }^{16}$ se puede explicar por esa falta de reacción:

Decían que daba pérdidas, pero este era un ferrocarril chico que no daba pérdidas reales, pero era quizás donde había menos protesta... Entonces arrancaron por acá, después Deseado, después Comodoro. ${ }^{17}$

16. Por ejemplo la línea de Puerto Deseado a Colonia Las Heras y Comodoro Rivadavia-Sarmiento fueron cerradas en 1978-1979, mientras que la de San Antonio Oeste y Jacobacci-Esquel, aunque con menor frecuencia y sin inversiones, siguieron funcionando.

17. Entrevista a Vicente Decaro, por Susana López, 28 de abril de 1988. 
Los diarios de la región anunciaron movilizaciones que luego no sucedieron. ${ }^{18}$ La dificultad que los trabajadores exhibieron para sostener su reclamo sin depender del apoyo de sectores no obreros (gobierno provincial, prensa o cámaras de comercio) presentan reacciones semejantes a las que veremos expresadas entre la mayoría de la dirigencia gremial, cuando se derrumbe el polo de desarrollo en los años 90.

\section{Inicio después de un final}

Es llamativa la ausencia de referencias al cierre de este ramal en varios trabajos sobre la huelga ferroviaria de 1961. Quizás la perspectiva de Fernando Ortega, quien defiende el proyecto de Frondizi, es la que sustenta la invisibilización del proceso. Este autor afirma que sólo se cerraron ramales "marginales" ${ }^{19} \mathrm{El}$ concepto de marginalidad se construye desde una mirada posicionada en la región metropolitana de Argentina, pero este ramal nada tenía de "marginal" para los trabajadores de Chubut.

A partir de allí la clase se estructuró en torno a nuevos núcleos obreros, especialmente textiles, metalúrgicos y otros sectores que surgian como actividades satélites al desarrollo industrial. Nuevos contingentes migrantes llegaron a la región, atraídos por la oferta de fuentes de trabajo; empezaba a tomar forma una clase obrera que tenía rasgos de novedad, pero también sostendría elementos de esa vieja clase.

A mediados de los 60 los obreros que arribaban para trabajar en las industrias comenzaron a desarrollar distintos procesos de lucha y organización. Para este apartado retomamos aportes de Binder, 2012; Gatica, 2007; y Fernández Picolo, Western y De Oto, 1990.

Durante 1966 hay movilizaciones de trabajadores de la construcción en reclamo de mejores salarios y del pago en término de los mismos, ante

18. "Ferrocarriles: El pueblo se moviliza en su defensa", titula el diario Jornada el sábado 24 de junio de 1961. Según el diario existía un movimiento para defender los amenazados tramos ferroviarios: "Obreros del riel, obreros en general, gobernantes, pueblo, partidos políticos, legisladores, se movilizan en defensa de un transporte que resulta indispensable en el sur". Se defiende la importancia estratégica del tren, pese a su ineficiencia en términos de costos, sosteniendo que son ferrocarriles de fomento, claves en una región a poblar como Patagonia: "La Patagonia recién ha comenzado a vivir la propia vida. Antes ha trabajado para el norte". El diario El Regional, de Gaiman, publica el 21 de junio de 1961: "Sería levantado el ramal ferroviario desde Puerto Madryn a Alto Las Plumas". Como bajada del título, sostiene "La medida ha de provocar gran repercusión. Habría movimiento en las fuerzas vivas".

19. "Solo se cerraron aproximadamente 1.000 kilómetros y la mayoría correspondía a ramales realmente marginales" (Ortega, 2010: 17). 
algunos cortes en las cadenas de pago del Estado provincial, ${ }^{20}$ y un conflicto en la textil Cualicrón por aumento salarial. La Asociación Obrera Textil (AOT) regional adhirió al primer paro general contra el gobierno de Onganía convocado por la CGT nacional, el 14 de diciembre de 1966.

En febrero de 1967 la CGT nacional definió un Plan de Lucha que planteaba la división del país en zonas, correspondiendo a Comodoro Rivadavia, Trelew y Río Gallegos la zona 14. El plan tuvo escaso impacto en la región, excepto una huelga de 24 horas de la Unión de Obreros de la Construcción de la República Argentina (UOCRA).

Recién tras la emergencia del Cordobazo y los Rosariazos, los trabajadores locales dieron muestras de una creciente actividad, aunque la dirigencia sindical seguiria sosteniendo una praxis colaboracionista con el gobierno y las patronales. La seccional regional de la CGT no adhirió a las medidas de fuerza, limitándose a un acompañamiento "moral y espiritual", postura que tomaron Empleados de Comercio, Municipales, Construcción y Gastronómicos (Jornada, 30 de mayo de 1969).

A partir de allí se inició una nueva etapa de conflictividad. Un sector de la clase obrera empezó a desarrollar acciones más contundentes: la UOCRA realizó una huelga solidaria con los trabajadores de El Chocón, en agosto de 1969. En 1970 ese gremio se sumó a las huelgas nacionales de abril, octubre y noviembre (convocadas por la CGT) e impulsó conflictos laborales contra diversas empresas locales.

La AOT se vio arrastrada a un largo conflicto por la presión de los trabajadores, pese a su explícita intención de sostener una práctica colaboracionista. Alrededor de 600 trabajadores fueron suspendidos por las textiles Tycora y Cualicrón, ubicadas en Puerto Madryn. Junto con Del Golfo eran fábricas pioneras, instaladas al amparo de los subsidios desde 1956. La AOT convocó una huelga con cierre de comercios junto a la Cámara de Industria, Comercio y Producción de Madryn, dándole al conflicto un carácter "regional" y planteando la defensa de la ciudad en tanto "corporación económica". ${ }^{21}$ Solicitaron que se garanticen los subsidios estatales para todas las empresas (Tycora y Cualicrón denunciaban haber sido "discriminadas"), reclamando créditos especiales para las empresas en conflicto.

20. Diario Jornada, 18 de noviembre de 1966, citado por Binder (2012).

21. Esto se hace evidente en el siguiente telegrama dirigido a Levingston, que es citado por Binder: "Asociación Obrera Textil Seccional Trelew interpreta su obligación hacer conocer al Excelentísimo señor Presidente de la Nación angustiosa situación aproximadamente seiscientas familias textiles por cierre establecimientos Cualicrón y Tycora de Puerto Madryn, solicitando respetuosamente su intervención personal para posibilitar solución urgente evitando no la quiebra de dos establecimientos, sino la de una ciudad patagónica", Jornada, 22 de septiembre de 1970 (subrayado de Axel Binder). 
La suspensión de los trabajadores continuaba, mientras la dirigencia sindical seguía el reclamo por vías formales, a través de notas a las autoridades. Finalmente las empresas cerraron sin que se produjeran conflictos en la calle. El Estado provincial otorgó 50 millones de pesos a la AOT, como subsidio para los obreros. ${ }^{22}$

También la UOCRA protagonizó diversos conflictos reclamando el respeto de las empresas a la legislación laboral, y el compromiso del Estado para evitar los abusos patronales. Denunciaban que las empresas no abonaban los salarios familiares, horas extras, días de enfermedad, aguinaldos y vacaciones.

Hacia fines de 1970 se comenzó a perfilar la construcción de una fracción del movimiento obrero que cuestionaba el "participacionismo" y avanzaba hacia posturas más combativas. Integrantes del gremio municipal, de la construcción, transporte, administración pública y agrupaciones textiles opositoras denunciaron la connivencia de los dirigentes sindicales de la CGT local con el gobierno.

La disputa se expresó en las elecciones de la AOT, entre el oficialismo de la lista "Granate" y el movimiento textil "Lealtad". La nómina de candidatos de estos últimos fue rechazada, argumentando problemas legales; los opositores denunciaron la elección como fraudulenta e ilegitima. En noviembre de 1970 también se produjeron elecciones en la CGT regional, evidenciándose nuevamente la división del movimiento obrero.

En la CGT se impuso el sector que planteaba una línea más combativa, dirigida por UOCRA y municipales. Pero esa elección fue anulada por la dirección nacional, encabezada por José Rucci, y sólo se permitió la presentación de la línea dirigida por Gilberto Hughes, ${ }^{23}$ de Luz y Fuerza, y Ayala, de la AOT.

También se venía impulsando la organización de nuevos sindicatos entre los estatales. El Sindicato de Obreros y Empleados de la Administración Pública (SOYEAP) se desarrolló en oposición al Estado provincial, que mantenía los salarios congelados desde 1966. En 1971 avanzaron en su organización los docentes, plegándose a huelgas nacionales desde el Centro de Maestros del Valle del Chubut y la Asociación de Docentes Provinciales. En marzo de 1972 nació el Sitravich (Sindicato de Trabajadores Viales del Chubut), un sindicato definido como "clasista" y cuyo funcionamiento se basaba en asambleas de base.

22. Por los cuales se produjeron denuncias de malversación contra el dirigente de la AOT, César Ayala.

23. Hughes, quien fuera dirigente sindical de la Unión Ferroviaria y la CGT en el período previo, ahora seguía siéndolo como afiliado de Luz y Fuerza. Ayala, de la AOT, se había formado junto a él y sería consecuente con su práctica colaboracionista. 


\section{La consolidación del modelo de polo de desarrollo}

En los siguientes años se consolidó el proyecto de industrialización subsidiada. Se conformaron dos tendencias dentro de la clase obrera, que se seguirian expresando en la conflictividad social durante las siguientes décadas. Una retomaba la praxis que vimos expresada en el marco del cierre del ferrocarril: buscar acuerdos con el gobierno, dirigir los reclamos ante éste, e intentar presentar las demandas sectoriales en clave regional, pretendiendo articularlas con sus respectivas patronales y las Cámaras de Comercio. Las alianzas buscaban ser construidas con sectores no obreros, y por ello las peticiones tendian a ser corporativas; los trabajadores de cada sector solicitaban subsidios junto a sus patronales, requiriendo para ello el acompañamiento del gobierno local.

Por otro lado se plantea una línea minoritaria, pero que siguió expresándose en diferentes momentos históricos, que proponia la necesidad de articular sus demandas a partir de la unidad entre diferentes fracciones de trabajadores. Apoyándose en la movilización y en medidas de confrontación directa, sus reclamos apuntaron contra las patronales. Esta tendencia centró sus demandas en clave laboral, antes que en la solicitud de subsidios a los empresarios, y por ello dichas exigencias podian trascender lo corporativo y transformarse en reclamos colectivos (aunque en escasas instancias esa potencialidad logró plasmarse en la realidad).

Durante 1972 esas perspectivas quedaron manifiestas. El traslado de presos políticos a la cárcel ubicada en Rawson generó la solidaridad de la población, entre los que se encontraban trabajadores y gremios. Se conformaron las Comisiones de Solidaridad con presos políticos, que darían asistencia a los detenidos y sus familiares. ${ }^{24}$ Tras la fuga de los principales líderes del PRT-ERP, Montoneros y FAR, el 15 de agosto de 1972 , se produjo el asesinato de 16 dirigentes de estos grupos a manos de la Armada en Trelew. El 11 de octubre las fuerzas represivas secuestraron a 16 militantes de la región, acusados de haber colaborado con la fuga; de inmediato se generó una gran movilización popular exigiendo su liberación, donde estaban presentes integrantes del SOYEAP, Sitravich, UOCRA y Municipales. La conducción de la CGT regional se opuso a este proceso de lucha, sin cuestionar el accionar de la dictadura.

Durante varios días se produjeron movilizaciones, y fue impactante la huelga general del 13 de octubre. El paro fue total en salud, comer-

24. Para conocer en profundidad estos hechos ver el citado trabajo de Fernández Picolo y otros. Alli se describe la polémica entre la CGT local y Agustín Tosco, que estaba preso en el penal de Rawson. 
cio, bancos, administración pública, estaciones de servicio, transporte, educación primaria, secundaria y superior. Se paralizaron las obras de construcción y las textiles cerraron sus puertas desde la mañana. Los obreros que construían la planta de aluminio finalizaron su jornada al mediodía. Nuevas huelgas generales se realizaron el 16, 20 y 27 de octubre, siendo enfrentadas por la CGT, pese a lo cual mantuvieron, en todos los casos, un impacto relevante. Hubo piquetes en algunas fábricas, registrándose enfrentamientos con la policía provincial.

Tras la apertura electoral, en 1973, la mayoría de los gremios se alinearon con el candidato a gobernador por el peronismo. A su vez se consolidó la división entre las principales orientaciones del movimiento obrero en la región: el sector que sustentaba una estrategia participacionista, alineada con el proyecto de polos de desarrollo; y el que buscaba mayor autonomía para los trabajadores, impulsando una propuesta que confrontaba con los sectores dominantes. El nuevo gobierno provincial profundiza el accionar represivo contra aquellas fracciones obreras que intentaban potenciar las luchas, creando cuerpos policiales especiales para atacar sus reclamos.

Esto se evidenció, por ejemplo, durante el conflicto que sostuvo el Sitravich a fines de 1973. En ocasión de realizar un corte del puente que cruza el Río Chubut en el marco de un paro por tiempo indeterminado, fueron reprimidos por la policía provincial: se trató de la primera acción de la recientemente creada "brigada antidisturbios". Finalmente, tras una amplia solidaridad de otros sindicatos no oficialistas, el Sitravich logró conseguir la mayoría de sus demandas. Esa solidaridad obrera volvió a manifestarse durante otro conflicto, el protagonizado por los docentes en mayo de 1974: allí el Sitravich convocó una huelga en apoyo a la Asociación de Docentes Provincial, y acompañó sus marchas junto a otros gremios.

El gobierno provincial y los sindicatos oficialistas atacaron estos procesos, generando listas de oposición en los sindicatos más combativos y persiguiendo laboralmente a algunos militantes. Para 1975 las articulaciones entre distintos colectivos obreros fueron quedando en el pasado, y comenzaba un cambio de signo. La práctica solidaria se vio reemplazada por una línea más corporativa en la consecución de sus reclamos.

Ese año ganó la conducción del Sitravich una dirigencia ligada al PJ provincial. En el marco de un nuevo conflicto docente (con los que antes habian sido solidarios) declararon no adherir "por encontrarse en positivas tratativas a nivel provincial y nacional" (Jornada, 12 de agosto de 1975). En cambio, sí apoyaron un reclamo, impulsado por el gobierno provincial, solicitando la continuidad de la promoción industrial: se observa el paso de una línea basada en la unidad de los trabajadores 
hacia otra que se sustentaba en la alianza con el gobierno y las patronales en nombre del "desarrollo de la Patagonia". ${ }^{25}$

La construcción de alianzas con las patronales con inversiones en la región y la sintonía en el discurso acerca de la necesidad de potenciar el desarrollo de Patagonia, fueron elementos clave a lo largo de la historia de esta clase. Si bien esta perspectiva parece robustecerse con la implantación de los polos de desarrollo, ya pueden verse elementos semejantes en los discursos y prácticas de los dirigentes sindicales de la etapa previa. En los testimonios de los cuadros sindicales del ferrocarril se repite que una de las claves de su accionar era la defensa de la "soberanía nacional", a partir de la centralidad del tren como factor de afianzamiento del Estado argentino en la región. Los tibios intentos de oponerse a su clausura se articularon en torno a este discurso.

Durante la dictadura, y en un marco de intensa vigilancia, se siguieron produciendo conflictos, en general vinculados a las condiciones de trabajo por planta. La iniciativa más evidente es la negativa a trabajar horas extras: hemos registrado este hecho entre los textiles de Trelew y en la planta de Aluar. La demanda de mejores condiciones de trabajo se realizaba a través de este método, que no contradecía la legalidad pero sí enfrentaba las "costumbres" de la región, donde se hacía necesario el uso intensivo de la aún escasa fuerza de trabajo disponible.

Tania Pérez Aguilar (2002) registra otra demanda, en este caso de un bono por productividad en la fábrica textil Supersil. Un importante conflicto se originó en la pesquera Ventura, de Madryn, en 1981, con la ocupación de la fábrica durante siete dias hasta conquistar mejores condiciones de trabajo, centradas en reivindicaciones de las trabajadoras mujeres.

A través de esos procesos de lucha se estructuraron comisiones internas en algunas textiles y en Aluar. También en la construcción comenzaron a elegirse delegados con una linea distinta al sindicato, que había asumido las posturas conciliadoras de su dirección nacional. Son esas experiencias de autoorganización las que generaron una acumulación de fuerza que se expresó tras el regreso al régimen constitucional. El fin de la dictadura no pareció alumbrar una clase obrera débil. En ese momento surgian nuevos agrupamientos sindicales y políticos, y se evidenciaba una mayor dinámica de los trabajadores. Adquirieron mayor fuerza varios agrupamientos políticos que se definían como de "izquierda" y que propusieron la organización de la clase obrera en términos de un proyecto político propio.

25. "Se tienden a robustecer las gestiones que se llevan a cabo en forma conjunta con otros gremios y el gobierno provincial, ante el poder ejecutivo nacional a fin de que se aporten los fondos necesarios" (Jornada, 13 de agosto de 1975). 
Las fuerzas que se habian acumulado en los años previos, y que estaban contenidas en el contexto represivo, parecieron liberarse para avanzar en nuevas conquistas. La intensa actividad politica y sindical que se manifestaba era evidencia de la acumulación de experiencias que venían desarrollando en la etapa previa de forma semiclandestina.

En la AOT, la Unión Obrera Metalúrgica (UOM) y la UOCRA se comenzó a debatir la necesidad de conformar listas que plantearan la disputa contra la conducción sindical. Las historias en la AOT y la UOM coinciden: en la elección de 1985 ganaron listas que se definian como pluralistas y combativas, siendo reemplazadas por las conducciones tradicionales en la siguiente elección.

En los casos en que las listas opositoras triunfaron, fueron procesos de amplia movilización social que luego no se tradujeron en una modificación profunda de los sindicatos. Si bien en los primeros años las nuevas direcciones plantearon cambios hacia una mayor democracia interna y presencia en las calles, pronto se fueron apartando de ese modelo. En la UOCRA la elección no pudo ganarse, en medio de denuncias de fraude por parte de la lista opositora.

La nueva conducción de la AOT se fue adaptando a las características de la dirigencia nacional. En sus testimonios los trabajadores destacaron la importancia que tuvo la presión de la conducción nacional a través del ahogo financiero a la regional cuando intentaba tomar posturas propias, y finalmente el proyecto se fracturó (Pérez Álvarez, 2011). La experiencia de ganar la regional de la UOM tampoco pudo consolidarse como un proyecto alternativo; en las siguientes elecciones el frente de 1985 se rompió, posibilitando la victoria de la línea que representaba la conducción nacional.

En esos años comenzaba a derrumbarse la promoción industrial a partir del avance del proyecto neoliberal. Se hicieron comunes los cierres de fábricas, produciéndose luchas como en la textil Gebco, que fue ocupada por sus obreros y culminó en una dura derrota, recordada en los años 90. Luego de 40 días de toma, en abril de 1987, la dirigencia sindical anunció el fin de la medida, destacando que habían logrado "arrancarle a la patronal el 80\% de las indemnizaciones", y manifestando su oposición "con quienes quieren utilizar esta lucha con fines sectarios y divisionistas". ${ }^{26}$ Se pretendía mostrar como una victoria un proceso que no había podido evitar el cierre de la fábrica, atacando a los grupos que planteaban una propuesta distinta a la conducción.

Los trabajadores de la región se encontraban ante un nuevo marco.

26. Jornada, 29 de abril de 1987. Se enfrentaba aquí a grupos textiles vinculados a partidos de izquierda, que habían cuestionado la falta de profundización de la lucha contra el cierre de la fábrica. 
Su historia de luchas pasaba por el reclamo de mejores condiciones laborales y de vender su fuerza de trabajo a mejor precio. La experiencia del ferrocarril no fue recuperada, o bien sólo lo fue en clave de llevar adelante una linea similar por parte de la mayoría de la clase. No había, aún, conciencia de que ahora la situación era diferente: en el marco de la estructura económica social que había conformado el neoliberalismo no sería fácil para los obreros despedidos reinsertarse en el mercado laboral.

El programa de la mayoría de los trabajadores (y de sus dirigencias) dejaba la lucha circunscripta a cada grupo de obreros, y en ese círculo sus intereses parecian igualarse con los de sus patrones. Según esa perspectiva no había otra forma de mantener el trabajo que no fuese defendiendo las empresas, exigiendo la continuidad de la promoción industrial y el otorgamiento de subsidios a sus dueños.

El año 1989 fue un punto de quiebre, donde se generaron las condiciones de realización hegemónica del neoliberalismo. Los trabajadores vieron arrasadas gran parte de sus organizaciones; en la región se aceleró la caída del polo de desarrollo y hubo gran cantidad de despidos. Las luchas reprodujeron la perspectiva de reclamar, a las diversas instancias del Estado, subsidios para sus patronales, limitándose a una exigencia centrada en la defensa "regional" que los constreñia a no trascender lo corporativo. ${ }^{27}$

\section{Reflexiones finales}

Todo proceso histórico se compone de cambios y continuidades, rupturas y permanencias. Es una tarea clave del historiador destacar cuánto hay de lo "viejo" en lo "nuevo", o cuánto de lo que aparece como novedad es realmente "novedoso". Esta línea de reflexión puede llevarnos a sostener que nada puede ser totalmente nuevo en la historia, ya que siempre lo que surge tiene vasos comunicantes con el pasado.

Pero esa perspectiva, correcta en líneas generales, subestima los momentos de quiebre en la historia, aquellos hechos que modifican la relación de fuerzas sociales. La integración de la Patagonia al mercado nacional en el contexto del proyecto de industrialización subsidiada marcó una ruptura. Se desarmaba, como parte del mismo proceso, un modelo de desarrollo previo de la región, que guardaba una relativa autonomía con respecto al mercado nacional. El ferrocarril era el eje de

27. En general la demanda específica era un subsidio para determinada empresa en crisis. De esta forma se hacía dificil articular hasta los reclamos de una misma rama económica, ya que cada colectivo obrero estaría luchando entre sí por acceder a una parte del limitado presupuesto del Estado. 
ese pequeño "mercado interno" articulado en la Patagonia argentina, que proveía de productos a una parte de las ciudades ubicadas más al sur.

La clase obrera sufrió allí una profunda transformación, aunque el avance de nuestra investigación nos muestra que ese cambio no fue total. Con la instalación del polo de desarrollo se configuró una nueva clase en términos estructurales, ya que en su mayoria provino de núcleos migrantes que se asentaron en la región durante las siguientes décadas. Pero esa clase, "nueva" en algunos aspectos, sostuvo diversas características de la "antigua" clase. Particularmente hemos visto que es notorio cómo se reprodujeron experiencias y tradiciones de la vieja clase obrera regional.

Se sostuvo la constante referencia a la "soberanía", planteando la defensa de políticas de excepción para la región, persuadidos de que era la única manera de asegurar el control del Estado sobre la Patagonia. En definitiva, se reprodujo el discurso del Estado argentino acerca de la centralidad de poblar la Patagonia en tanto región rica en recursos naturales y supuestamente amenazada por otros paises, que fue clave en la legitimación de la "conquista del desierto" y de los hechos de represión contra huelgas en el sur del país (ver el ejemplo clásico en Bayer, 1974). Un sector de los trabajadores hacía suyos estos argumentos, usándolos como un recurso que serviría para legitimar la continuidad de las fuentes de trabajo en la región.

Esa perspectiva generaba costos para los obreros: sólo era posible articular la misma sintonía discursiva del Estado si las demandas se expresaban en términos regionales, y no como exigencias de la clase operaria. Para que la petición tomase carácter "regional" debía ser apoyada por el gobierno local, los empresarios del rubro o algún sector afin. Los trabajadores quedaban restringidos a canalizar sus reivindicaciones a partir de alianzas con sus respectivos patrones, inhibiendo la posibilidad de vincular sus reclamos con el resto de su clase.

Cuando los sectores no obreros consideraban que ya no era necesaria la continuidad de determinadas actividades económicas en la región, como sucedió con el ferrocarril en el 60 y volvió a ocurrir en los 90 con las industrias, los trabajadores quedaron solos y casi sin herramientas para articular respuestas más contundentes. La "nueva" clase obrera se encontró, ante el desmoronamiento del polo de desarrollo, con similares dificultades para articular una propuesta que pudiese preservar su fuente de empleo a la que había evidenciado la "vieja" clase frente al cierre del ferrocarril. 


\section{Referencias bibliográficas}

Accorinti, Vicente y otros (1989), Los ferroviarios que perdimos el tren, Trelew: Secretaria de Cultura de la Nación.

Bandieri, Susana (2005), Historia de la Patagonia, Buenos Aires: Sudamericana.

-, Graciela Blanco y Gladys Varela (dirs.) (2006), Hecho en Patagonia. La historia en perspectiva regional, Neuquén: Educo.

Bayer, Osvaldo (1974), Los vengadores de la Patagonia trágica, tomos I, II y III, Buenos Aires: Galerna; tomo IV, Frankfurt: Hammer, 1978.

Binder, Axel (2012), Crónica de una protesta anunciada: conflictividad regional y nacional a través de la prensa del noreste de Chubut (Diario Jornada, 1966-1971), tesis de Licenciatura en Historia, UNPSJB.

Cena, Juan Carlos (2009), Ferroviarios, sinfonía de acero y lucha, Buenos Aires: Monarefa-La Nave de los Locos.

Fernández Picolo, Mauricio, Wilda Western y Alejandro De Oto (1990), Autoritarismo y participación popular: Trelew, octubre de 1972, tesis de Licenciatura en Historia, UNP, Sede Trelew.

Gatica, Mónica (1998), “Trelew, ¿un polo de desarrollo y modernización?”, ponencia en IV Jornadas de Historia, Caleta Olivia, UNPA.

- (2007), Hacedores de caminos, Buenos Aires: Imago Mundi.

- y G. Pérez Álvarez (2012), "No solamente pasaba el viento: sindicatos, huelgas, boicots, cortes de vías y lucha politica en los primeros pasos del movimiento obrero en el noreste del Chubut (1917-1922)", en Arias Bucciarelli (dir.), Diez territorios nacionales y catorce provincias, Argentina, 1860-1955, Buenos Aires: Prometeo, pp. 187-214.

Jones, Lewis (1966), Una nueva Gales en Sudamérica, Trelew: Comisión del Centenario.

Jones, Matthew (1981), Trelew, un desafio patagónico, tomo I: 1886-1903, Puerto Madryn: Dirección de Cultura.

Lucita, Eduardo (1999), La patria en el riel. Un siglo de lucha de los trabajadores ferroviarios, Buenos Aires: Pensamiento Nacional.

Ortega, Fernando Ariel (2010), "De la modernización a la racionalización. Políticas adoptadas por Frondizi ante la crisis ferroviaria (1958-1962)", enH-industri@, año 4, nº 7.

Pérez Aguilar, Tania (2002), Avance de investigación, Taller V, del Área VI de la Licenciatura en Historia, Facultad de Humanidades y Ciencias Sociales, UNP, sede Trelew.

Pérez Álvarez, Gonzalo (2011) "Con hilos rotos vamos tejiendo otra historia. Lucha y experiencia obrera en el parque textil de Trelew", Sociohistórica $\mathrm{n}^{\circ}$ 27, La Plata: FAHCE-UNLP, pp. 13-39.

- (2013) Patagonia, conflictividad social y neoliberalismo. El noreste de Chubut (1990-2005), Buenos Aires: Imago Mundi

- (2015), "A Study on the Contextual Features that Shaped the Formation of a Working Class Fraction: the Workers of Northeast Chubut (Patagonia, 
Argentina)", Workers of the World - International Journal on Strikes and Social Conflict, vol. 1, n 6, Lisboa, pp. 84-104.

Perroux, François (1955), "Notes sur la notion de pole de croissance", Economie Appliquée, $\mathrm{n}^{\circ}$ 8, enero-junio.

Rougier, Marcelo (dir.) (2007), Politicas de promoción y estrategias empresariales en la industria argentina, 1950-1980, Buenos Aires: Cooperativas.

Schneider, Alejandro (2006), Los compañeros. Trabajadores, izquierda y peronismo. 1955-1973, Buenos Aires: Imago Mundi.

Scodeller, Gabriela (2007), "La huelga ferroviaria de 1961 en la provincia de Mendoza", Actas Primeras Jornadas Nacionales de Historia Social, La Falda.

Thompson, Edward Palmer (1989), La formación de la clase obrera en Inglaterra, Barcelona: Crítica.

$$
* * *
$$

Titulo: "Old" and "new" working class in Patagonia Argentina: from the beginning of the railway to the rise of state-subsidized industries

Resumen: Desde 1956 el Estado nacional de Argentina promovió la instalación de industrias en la Patagonia, modificando una estructura económica que hasta alli tenía como eje articulador el ferrocarril, el cual fue clausurado en 1961. Hacia mediados de los años 70 la región había sido transformada, a partir de la instalación de un polo textil sintético, la única planta productora de aluminio primario de Argentina y la llegada de migrantes atraídos por la oferta de puestos de trabajos. Indagamos este momento de quiebre, preguntándonos específicamente por la situación de la clase obrera. Para ello exploramos su historia, la destrucción y conformación de organizaciones obreras, el desarrollo de nuevos núcleos obreros y los vínculos con las experiencias y tradiciones de los antiguos núcleos.

Palabras clave: Clase obrera - Patagonia argentina - Ferrocarril - Industrias

Abstract: From 1956 the national State of Argentina promoted the installation of industries in the Patagonia. This way modified his economic structure which until then had like axis railroad, which was closed in 1961. About middle of the years 70 , the region had been transformed, from the installation of a textile synthetic pole, the only producing plant of primary aluminium of Argentina and the arrival of migrants attracted by the offer of positions of works. We investigate this moment of break, wondering specifically for the situation of the working class. For it we explore his history, the destruction and conformation of working organizations, the development of new working cores and the links with the experiences and traditions of the former cores.

Keywords: Working class - Patagonia Argentina - Railway - Industries

Recepción: 6 de marzo de 2015. Aprobación: 11 de julio de 2015 